\title{
An Approach to Measuring the Achievement or Proficiency of an Examinee
}

\author{
Rand R. Wilcox \\ Center for the Study of Evaluation \\ University of California at Los Angeles
}

\begin{abstract}
Various school systems are developing proficiency tests which are conceptualized as representing a variety of skills with one or more items per skill. This paper discusses how certain recent technical advances might be extended to examine these tests. In contrast to previous analyses, errors at the item
\end{abstract}

level are included; and it is shown that inclusion of these errors implies that a substantially longer test might be needed. One approach to this problem is described, and directions for future research are suggested.

Throughout the United States efforts are being made to develop tests to measure the proficiency of students attending the local schools. These tests are used to determine whether a student will be awarded a high school diploma and to decide whether an examinee should be advanced to the next grade level. The tests are sometimes conceptualized and constructed as follows: First, a group of teachers, parents, content experts, and other interested persons work together to identify those skills believed to be a basic part of a student's education. For example, interest may focus on competency in mathematics, in which case the skills might include addition, subtraction, computing percentages, and so forth. Corresponding to each skill, test items are constructed for the purpose of determining whether an examinee has acquired the skill in question. Here it is assumed that these test items have been examined for any ambiguities or misrepresentations and that appropriate corrections have been made when necessary.

Because of the large number of skills that have been identified, it is impractical to test an examinee on every one. Accordingly, a random sample of skills is used to make inferences about the proportion of skills that an examinee has acquired. The test administered to an examinee consists of items that represent the skills. Decisions concerning proficiency are made according to some predetermined passing score. For example, a requirement for receiving a high school diploma might include taking a mathematics test and successfully answering $70 \%$ of the items or demonstrating mastery of $70 \%$ of the skills. Note that these two decisions are not necessarily equivalent. As a simple illustration, imagine a test of 10 skills with 3 items per skill for a total of 30 items. Further suppose that a mastery decision is made for a particular skill if the examinee responds correctly to two out of the three corresponding items. In other words, an allowance is being made for the possibility that an

(C) Copyright 1980 West Publishing Co. 
examinee has acquired the skill but gives an incorrect response because of some distraction or carelessness. In this case it is possible (but perhaps unlikely) that an examinee will get less than $70 \%$ of the items correct yet demonstrate mastery of more than $70 \%$ of the skills.

The purpose of this paper is to demonstrate how certain recent technical advances can be extended and applied to the type of test described above. Emphasis is given to the problem of determining how many skills to include on a test. As will become evident, the analysis has implications about how many items to use per skill. In the case of multiple-choice test items, there are also possible implications about the number and quality of the distractors that are being used.

The situation considered here is similar to a common conceptualization of a mastery test. A mastery test is frequently regarded as consisting of items randomly sampled from some larger item pool (e.g., Harris, 1974; Huynh, 1976; Novick \& Lewis, 1974; Wilcox, 1977). The item domain might exist de facto or it might be a convenient conceptualization. Based on this "item sampling" view, the binomial error model (Lord \& Novick, 1968, chap. 23) is then used to describe the observed responses of the examinees. In particular, the probability function of $x$, the observed (number-correct) score of an examinee, is given by

$$
f(x \mid p)=\left(\begin{array}{l}
n \\
x
\end{array}\right) p^{x}(1-p)^{n-x}
$$

where $p$ is referred to as the examinee's percent-correct true score. The goal of the test is to determine whether $p$ is above or below a known constant $p_{0}$. The main difference between mastery tests and the present situation is that here the view is taken that skills, not items, are being sampled and that there might be more than one item per skill. Moreover, the analysis given here includes errors at the item level, whereas for the binomial error model these errors are ignored. (For the case in which only one skill is being examined in terms of a population of examinees, the reader is referred to Macready and Dayton, 1977.)

Let $\zeta$ be the proportion of skills that an examinee knows. Consistent with the approach to mastery tests, it is assumed that the goal of a proficiency test is to determine whether $\zeta$ is above or below a known constant, $\zeta_{0}$. Before describing the main results of solving this problem, a more precise description of the framework of the problem will be given.

\section{Some Definitions}

Consider a specific randomly selected skill and let $k$ be the number of items used to determine mastery of this skill. For each of these $k$ items it is assumed that an examineee who has mastered the skill might give an incorrect response because of a momentary distraction, carelessness, and so forth. Let $\alpha_{i}(i=1, \ldots, k)$ be the probability of this event for the $i^{\text {th }}$ item. In a similar manner, let $\beta_{i}$ be the probability of not knowing and guessing the correct response to the $i^{\text {th }}$ item. Note that $\alpha_{i}$ and $\beta_{i}$ are both conditional probabilities. Finally, a mastery decision is made for the skill if $y$, the number correct out of the $k$ items associated with the skill, is greater than or equal to a specified passing score $y_{0}$.

The framework described above is similar to a number of models proposed by various authors to describe tests (e.g., Brownless \& Keats, 1958; Knapp, 1977; Macready \& Dayton, 1977; Marks \& Noll, 1967; Wilcox, 1979b). Macready and Dayton (1977, p. 100) imply that their model is appropriate when mastery of a skill is an all-or-none process. However, as noted by Wilcox (1979b), this does not mean that an all-or-none view of learning is required in order to use their model. Macready and Dayton use a more general family of decision rules for determining mastery of a particular skill. Their decision rule is defined in terms of a particular skill and a population of examinees, whereas 
here, at least for the moment, the emphasis is on making a decision for a specific examinee in terms of a particular randomly selected skill. It is readily seen, therefore, that their decision rule does not apply to the present situation.

Finally, let the vector $y=\left(y_{1}, \ldots, y_{k}\right)$ be a sequence of " 1 's" and " 0 's" designating a particular response pattern of "corrects" and "incorrects" on the $k$ items where a " 1 " means a correct and a " 0 " an incorrect response.

Based on the above definitions, and for the assumption of local independence (Lord \& Novick, 1968 , section 16.3), it follows that the probability of a mastery decision for the skill is

$$
\begin{aligned}
\operatorname{Pr}(y & \geq y_{0} \mid \text { mastery of the skill) } \\
& =\xi_{1} \text { (say) } \\
& =\sum_{y: y \geq y_{0}} \prod_{i=1}^{k}\left(1-\alpha_{i}\right)^{y_{i}} \alpha_{i} 1-y_{i}
\end{aligned}
$$

where the summation is over all vectors $\underline{y}$ such that $y \geqslant y_{0}$. In addition,

$$
\begin{aligned}
\operatorname{Pr}(y & \geq y_{0} \mid \text { nonmastery of the ski1l) } \\
& =\xi_{2} \text { (say) } \\
& =\sum_{y: y \geq y_{0}} \prod_{i=1}^{k} \beta_{i}^{y_{i}}\left(1-\beta_{i}\right)^{1-y_{i}} .
\end{aligned}
$$

If, as in Macready and Dayton's Model II, it is assumed that $\alpha_{i}=\alpha$ and $\beta_{i}=\beta$ for $i=1, \ldots, \mathrm{k}$, then Equations 2 and 3 take on the more familiar form of the binomial probability function, namely,

$$
\xi_{1}=\sum_{y=y_{0}}^{k}\left(\begin{array}{l}
k \\
y
\end{array}\right)(1-\alpha)^{y} \alpha^{k-y}
$$

and

$$
\xi_{2}=\sum_{y=y_{0}}^{k}\left(\begin{array}{l}
k \\
y
\end{array}\right) \beta^{y}(1-\beta)^{k-y} .
$$

\section{A Conservative Solution to the Problem of Determining the Number of Skills to Include on the Test}

Thus far it has been merely the ground work for handling certain technical problems associated with so-called proficiency tests that has been laid. In this section the determination of how many skills 
to include on the test is considered. The analysis is made in terms of a single examinee.

For a randomly selected skill, the probability of a mastery decision is

$\gamma=\xi_{1} \zeta+\xi_{2}(l-\zeta)$.

Thus, the probability of $x$ mastery decisions among $n$ randomly selected skills is

$\left(\begin{array}{l}n \\ x\end{array}\right) \gamma^{x}(1-\gamma)^{n-x}$.

Let $x_{0}$ be the passing score for the test. In other words, the decision $\zeta \geqslant \xi_{0}$ is made if $x \geqslant x_{0}$; if $x<x_{0}$, the reverse is said to be true. Here it is assumed that $x_{0}$ is the smallest integer such that $x_{0} / n \geqslant \zeta_{0}$.

The goal is to find a conservative solution to the choice for $n$. In particular it is desirable to choose the smallest $n$ so that the probability of a correct decision (CD) is reasonably close to 1 , regardless of the actual value of $\zeta$. To solve this problem it is necessary for the investigator to specify an additional constant, $d^{*}>0$. The idea is that if $\zeta \leqslant \zeta_{0}-\delta^{*}$ or $\zeta \geqslant \zeta_{0}+\delta^{*}$, it is desirable to choose the smallest $n$ so that

$\operatorname{Pr}(C D) \geq \mathrm{P}^{\star}, 1 / 2<\mathrm{P}^{\star}<1$.

If, however, $\zeta_{0}-\delta^{*}<\zeta<\zeta_{0}+\delta^{*}$, either decision is said to be correct. The open interval $\left(\zeta_{0}-\delta^{*}, \zeta_{0}+\right.$ $\left.\delta^{*}\right)$ is called the indifference zone. The situation is similar to the one considered by Fhanér (1974) and Wilcox (1979a). Here, however, the errors represented by the probabilities $\alpha_{i}$ and $\beta_{i}$ associated with each skill are taken into account. Note that if $\delta^{*}=0$, it may be impossible to find an $n$ that satisfies Equation $\mathbf{8}$ for all possible values of $\xi$. For a more extensive discussion of the indifference zone approach to statistical problems (including the choice of $\delta *$ ), the reader is referred to Gibbons, Olkin, and Sobel (1977). Further comments on the choice of $\delta^{*}$ are made below. In particular, it is shown that $\delta^{*}>0$ is a necessary but not a sufficient condition for solving the problem at hand.

Observe that if $\zeta<\xi_{0}$, the probability of a correct decision $[\operatorname{Pr}(C D)]$ is given by

$\sum_{x=0}^{x_{0}-1}\left(\begin{array}{l}n \\ x\end{array}\right) \gamma^{x}(1-\gamma)^{n-x}$

and if $\zeta \geqslant \zeta_{0}$, the $\operatorname{Pr}(C D)$ is equal to

$\sum_{x=x_{0}}^{n}\left(\begin{array}{l}n \\ x\end{array}\right) \gamma^{x}(1-\gamma)^{n-x}$.

Moreover, Equation 9 is a decreasing function of $\gamma$ and Equation 10 increases as $\gamma$ gets large (Fhanér, 1974). Since $\gamma=\xi_{1} \xi+\xi_{2}(1-\xi)$, it follows that $\gamma$ is an increasing function of $\xi$ if $\xi_{1}>\xi_{2}$. A situation in which $\xi_{1} \leqslant \xi_{2}$ would seem to be highly unusual, and so $\xi_{1}>\xi_{2}$ is assumed throughout.

Consider the case $\zeta \geqslant \zeta_{0}+\delta^{*}$. To ensure that Equation 10 is greater than or equal to $P^{*}$ for any $\zeta$, it is sufficient to consider the value of $\zeta$ for which it is a minimum. From the above discussion, it follows that this value is $\zeta=\zeta+\delta^{*}$. From Fhanér (1974) it can be seen that it is always possible to choose an $n$ satisfying Equation 8 if $\gamma>\zeta_{0}$. In terms of $\delta^{*}$ this means that a sufficient condition for being able to find an $n$ satisfying Equation 8 is to have 


$$
\delta^{*}>\frac{\zeta_{0}{ }^{-\xi_{2}}}{\xi_{1}-\xi_{2}}-\zeta_{0} .
$$

Note that for the binomial error model used by Fhanér (1974), $\xi_{1}=1$ and $\xi_{2}=0$, in which case the requirement given by Equation 11 is $\delta^{*}>0$.

Next the effect of $\xi_{1}$ and $\xi_{2}$ on the $\operatorname{Pr}(C D)$ for $\xi \geqslant \zeta_{0}+\delta^{*}$ is considered. From the above results it is readily seen that the $\operatorname{Pr}(\mathrm{CD})$ is minimized when $\xi=\xi_{0}+\delta^{*}$, regardless of the values for $\xi_{1}$ and $\xi_{2}$. Furthermore, $\gamma$ is an increasing function of both $\xi_{1}$ and $\xi_{2}$. Thus, to find a conservative solution to the choice of $n$ (i.e., an $n$ that satisfies Equation 8 regardless of the value of $\xi_{1}$ or $\xi_{2}$ ), lower bounds to both $\xi_{1}$ and $\xi_{2}$ are needed. Here it is assumed that there is no data available for estimating $\xi_{1}$ and $\xi_{2}$. Thus, the investigator must specify (using nonstatistical techniques) lower bounds to $\xi_{1}$ and $\xi_{2}$ that are consistent with the types of items being used. In practice this might be done by specifying an upper bound to $\alpha$ and a lower bound $\beta$ and using Equations 4 and 5. This is illustrated below.

For $\zeta \leqslant \zeta_{0}-\delta^{*}$ it can be seen that $\gamma<\zeta_{0}$ is required, which implies that

$$
\delta^{*}>\zeta_{0}-\frac{\zeta_{0}-\xi_{2}}{\xi_{1}-\xi_{2}} \text {. }
$$

In summary, it can be guaranteed that the probability of a correct decision is at least $P^{*}$, if Equations 11 and 12 are satisfied, by choosing the smallest $n$ so that both Equations 9 and 10 are greater than or equal to $P^{*}$. As for $\xi_{1}$ and $\xi_{2}$ this time $\xi$ is set to $\xi_{0}-\delta^{*}$ and upper bounds to these two quantities are used. In contrast to the case $\zeta \geqslant \zeta_{0}+\delta^{*}$, this might be accomplished by specifying a lower bound to $\alpha$ and an upper bound to $\beta$ and again using Equations 4 and 5.

\section{Examples}

\section{An Illustration with $k=1$}

Consider a situation in which a single item, $k=1$, is used to measure each skill and suppose that $\xi_{0}=.8, \delta^{*}=.1$, and $P^{*}=.90$. For this special case $\xi_{1}=1-\alpha$ and $\xi_{2}=\beta$ (assuming, of course, $y_{0}=1$ ). Consider the case $\zeta \leqslant \zeta_{0}-\delta^{*}$. As previously explained, the $\operatorname{Pr}(C D)$ given by Equation 9 is minimized at $\zeta=\zeta_{0}+\delta^{*}$. Since the value of $\xi_{1}$ and $\xi_{2}$ are unknown, Equation 9 cannot be evaluated. Suppose, however, that multiple-choice test items are being used with three distractors per item. For the sake of illustration it is assumed that the highest possible value of $\xi_{2}$ (the probability of guessing) is .4. If the test items are at all reasonably constructed, it would be expected that $\xi_{2}$ has a smaller value than .4. However, the exact value of $\xi_{2}$ is unknown and so to be conservative the case $\xi_{2}=.4$ is considered. For similar reasons it is assumed that $\alpha \geqslant 0$ and so the case $\alpha=0$ is considered implying that $\xi_{1}=1$.

With $\xi_{1}=1$ and $\xi_{2}=.4$, Equation 12 says that to be certain that an $n$ can be found so that $\operatorname{Pr}(C D)$ $\geqslant P^{*}$ it must be that $\delta^{*} \geqslant .133$. Thus, if $\delta^{*}>.1$ is judged to be unacceptable, steps must be taken to decrease the upper bound to $\xi_{2}$. For example, if the number of distractors is increased to four or $\xi_{2} \leqslant$ .3 , say, in which case the inequality in Equation 12 becomes $\delta * \geqslant .033$. Henceforth, it is assumed that $.15 \leqslant \beta \leqslant .3$. Since $\delta^{*}$ was chosen to be .1 , it is certain that an $n$ exists satisfying the desired probability guarantee.

With $\zeta=\xi_{0}+\delta^{*}=.9$, the $\operatorname{Pr}(\mathrm{CD})$ is minimized by setting $\xi_{1}=1-\alpha=.9$ and $\xi_{2}=\beta=.15$. In this case, $\gamma=.825$ and so 
$\operatorname{Pr}(C D)=\sum_{x=x_{0}}^{n}\left(\begin{array}{l}n \\ x\end{array}\right) \cdot 825^{x} \cdot 175^{n-x}$.

For $\xi \leqslant \xi_{0}-\delta^{*}=.7$, the minimum probability of a correct decision occurs at $\xi=.7$. In terms of $\xi_{1}$ and $\xi_{2}$, set $\alpha=0$ and $\beta=.3$, so $\xi_{1}=1, \xi_{2}=.3, \gamma=.79$, and

$\operatorname{Pr}(C D)=\sum_{x=x 0^{x}}^{x_{0}}\left(\begin{array}{l}n \\ x\end{array}\right) \cdot 79^{x} \cdot 21^{n-x}$.

From Wilcox (1979a) it follows that the smallest $n$, so that Equation 10 has a value of at least $P^{*}=$ .9 , is given approximately by

$n=\lambda^{2} \zeta_{0}\left(1-\zeta_{0}\right) /\left(\gamma_{1}-\zeta_{0}\right)^{2}$

where $\lambda$ is the $P^{*}$ quantile of the standard normal distribution and $\gamma_{1}$ is the value of $\gamma$ when $\zeta=\zeta_{0}+$ $\delta^{*}$. With $\xi_{1}=.9$ and $\xi_{2}=.15$,

$$
\begin{aligned}
& \approx \\
& (1.28)^{2}(.8)(.2) /(.825-.8)^{2} \\
& =419 .
\end{aligned}
$$

As for Equation 11 the smallest $n$ is given approximately by

$$
\lambda^{2} \zeta_{0}\left(1-\zeta_{0}\right) /\left(\zeta_{0}-\gamma_{2}\right)^{2}
$$

where $\gamma_{2}$ is the value of $y$ when $\zeta=\zeta_{0}-\delta^{*}$. In the illustration in this paper $n \approx 2621$. Thus, $n=2621$ skills would be used.

It is evident that for practical purposes, $n=2621$ is un acceptable. Suppose instead there are completion items, in which case guessing is virtually ruled out. For illustrative purposes, suppose $\beta=0$, which appears to be approximately true for the test data examined by Macready and Dayton (1977) and that $0 \leqslant \alpha \leqslant .02$. In this case $\gamma_{1}=.882, \gamma_{2}=.7$, and $n \approx 39$. If $0 \leqslant \alpha \leqslant .05, \gamma_{1}=.855, \gamma_{2}=.7$, and $n \approx 87$. If errors at the item level (i.e., $\xi_{1}=1$ and $\xi_{2}=0$ ) are ignored, the resulting value of $n$ is approximately 29 .

\section{An Illustration with $k=3$}

The second illustration is the same as the first except that it is assumed that there are $k=3$ items per skill. The primary purpose of this illustration is to see how much the required number of items can be reduced by increasing $k$. As before, it is assumed that $.15 \leqslant \beta \leqslant .3$ and $0 \leqslant \alpha \leqslant .1$.

With $\alpha=.1$ and $\beta=.3$, and with a mastery decision for a particular skill made when the examinee gets at least 2 of the 3 items correct (i.e., $y_{0}=2$ ), Equations 3 and 4 yield $\xi_{1}=.972$ and $\xi_{2}=.216$. When $\alpha=0$ and $\beta=.15, \xi_{1}=1$, and $\xi_{2}=.06$. Thus, for $\xi=\zeta_{0}-\delta^{*}, \xi_{1}=1$ and $\xi_{2}=.216$ are used, implying that $\gamma=.7648$. Hence, 
$\operatorname{Pr}(C D)=\sum_{x=0}^{x_{0}-1}\left(\begin{array}{l}n \\ x\end{array}\right) \cdot .7648^{x}(.2352)^{n-x}$.

As for $\zeta=\zeta_{0}+\delta^{*}, \gamma=.88$, and

$$
\operatorname{Pr}(C D)=\sum_{x=x_{0}}^{n}\left(\begin{array}{l}
n \\
x
\end{array}\right) \cdot 88^{x} \cdot 12^{n-x} .
$$

It follows that the smallest number of skills required is approximately $n=212$. The exact value was calculated on an IBM 360/91 computer and found to be $n=219$. Thus, the total number of items is decreased considerably, but over 600 items would still be needed on the test.

\section{An Illustration with \\ Tighter Bounds on $\alpha$ and $\beta$}

To illustrate the effect of having tighter bounds on $\alpha$ and $\beta$, suppose that $.0 \leqslant \alpha \leqslant .02$ and $.2 \leqslant \beta \leqslant$ .3 and set $y_{0}=3$. Otherwise the situation is assumed to be the same as in the previous illustration. In this case $\gamma=.848$ when $\xi=.9, \xi_{1}=.941$, and $\xi_{2}=.008$. Also, $\gamma=.7027$ when $\zeta=.7, \xi_{1}=1$, and $\xi_{2}=$ .027 . It follows that the minimum $n$ required is approximately 114 . Thus, to guarantee that the probability of a correct decision is at least .9 , a total of $3(114)=342$ items would be used.

\section{Retrospective Studies Using Latent Structure Models}

The illustrations in the previous section demonstrate rather dramatically that including errors at the item level might have a substantial effect on the number of items used on the test. Moreover, even with "tight" bounds on the parameters $\alpha$ and $\beta$, an extremely large number of items might be required. Several approaches to this problem might be used. For example, there might be a more optimal choice for $k$, the number of items per skill. In the case of multiple-choice items, increasing the number of distractors (cf. Lord, 1977) might be considered. In this section still another approach based on latent structure models is outlined. The approach represents a slight extension of one used by Wilcox (1979c).

In contrast to the earlier sections of the paper, it is now assumed that data exist for a random sample of $N$ examinees who have taken a test consisting of $n$ skills with $k \geqslant 3$ items per skill. The reason for the restriction on $k$ is explained below. An additional difference from previous sections is that the accuracy of the test is examined in terms of comparing $\zeta$ to $\zeta_{0}$ for the typical or "average" examinee among those being tested. This alternative perspective does not affect the results previously described. If an examinee's true score is close to $\zeta_{0}$, an extremely large number of items might be needed to accurately determine whether $\zeta$ is above or below $\zeta_{0}$. In some situations an investigator might also be interested in the accuracy of a test in terms of a population of examinees, for example, all the students attempting to graduate from high school. It may be that most examinees have a true score that is not close to $\zeta_{0}$ or perhaps most true scores fall within the indifference zone, in which case the test is usually giving accurate results. This section outlines how existing results on latent structure models can be used to detect this situation. 
Firstly, it is observed that for an examinee responding to $k \geqslant 3$ items per skill for a total of $n$ skills, it is possible to use latent structure models (e.g., Anderson, 1954; Formann, 1978; Goodman, 1974; Green, 1951; Harper, 1972; Lazarsfeld \& Henry, 1968) to estimate $\beta_{i}$, the probability of guessing the $i^{\text {th }}$ item among the $k$ items of a randomly sampled skill, $\alpha_{i}$ the probability of "forgetting" the $i^{\text {th }}$ item, and $\zeta$. An illustration of an iterative approximation to the maximum likelihood estimator is given by Macready and Dayton (1977). Note that the role of item and examinee is reversed in the paper by Macready and Dayton. Here the parameters $\alpha_{i}, \beta_{i}$, and $\xi$ are defined in terms of a single examinee and a domain of skills, whereas Macready and Dayton define them in terms of a single skill and a population of examinees. However, the estimation procedure for the present situation is essentially the same, so it is not discussed further except to say that initial estimates are available from Wil$\operatorname{cox}(1979 b)$.

For the $j^{\text {th }}$ examinee, let $\hat{\xi}_{j}$ be the resulting estimate of $\zeta$. Define

$\hat{u}=N^{-1} \sum_{j} \hat{\zeta}$

$\hat{\mu}_{1}=N^{-1} \sum_{j} \hat{\zeta}^{2}$

and

$$
\hat{\sigma}^{2}=\hat{\mu}_{1}-(\hat{\mu})^{2} \text {. }
$$

For the reasons given by Wilcox (1979b), $\hat{\mu}$ and $\hat{\sigma}^{2}$ may be used to estimate the mean, $\mu$, and variance, $\sigma^{2}$, of the true score distribution.

Let

$$
\begin{aligned}
\tau_{1} & =\mu, \text { if } \mu<\zeta_{0}-\delta^{*} \\
& =\zeta_{0}-\delta^{*} \text {, if } \zeta_{0}-\delta^{*} \leq \mu \leq 1
\end{aligned}
$$

$m_{1}=\max \left[\mu\left(\zeta_{0}-\delta^{*}-\mu\right),\left(\mu-\zeta_{0}+\delta^{*}\right)(1-\mu)\right]$

$$
\begin{aligned}
\phi_{1} & =\frac{\sigma^{2}}{\sigma^{2}+\left(\tau \tau_{1}-\mu\right)^{2}}, \text { if } 0<\sigma^{2} \leq m_{1} \\
& =\left(\mu(1-\mu)-\sigma^{2}\right) /\left(1-\tau_{0}+\delta^{*}\right)\left(\zeta_{0}-\delta^{*}\right) \text {, otherwise }
\end{aligned}
$$




$$
\begin{aligned}
m_{2} & =\max \left[\mu\left(\zeta_{0}+\delta^{\star}-\mu\right),\left(\mu-\zeta_{0}-\delta^{*}\right)(1-\mu)\right] \\
\tau_{2} & =\zeta_{0}+\delta^{*}, \text { if } \mu<\zeta_{0}+\delta^{*} \\
& =\mu, \text { if } \zeta_{0}+\delta^{*} \leq \mu \leq 1 \\
\phi_{2} & =\frac{\sigma^{2}}{\sigma^{2}+\left(\tau 2^{-\mu)^{2}}\right.}, \text { if } 0<\sigma^{2} \leq m_{2} \\
& =\left(\mu(1-\mu)-\sigma^{2}\right) /\left(1-\zeta_{0}-\delta^{*}\right)\left(\zeta_{0}+\delta^{*}\right), \text { otherwise. }
\end{aligned}
$$

Following Wilcox (1979c), results reported by Skibinsky (1977) can be applied to show that for $\varepsilon_{1}=\operatorname{Pr}$ $\left(x \geqslant x_{0}, \zeta \leqslant \zeta_{0}\right)$, the probability of a false-positive decision, there is the inequality

$$
\varepsilon_{1} \leq \phi_{1} \sum_{x=x_{0}}^{n}\left(\begin{array}{l}
n \\
x
\end{array}\right) \gamma_{1}^{x}\left(1-\gamma_{1}\right)^{n-x}
$$

where $\gamma_{1}$ is the value of $\gamma$ when $\zeta=\zeta_{0}+\delta^{*}$. As in the previous section, it is assumed that for a specific examinee, the probability of getting $x$ mastery decisions is given by the binomial probability function (cf. Lord \& Novick, chap. 23). As for the probability of a false-negative decision, say $\varepsilon_{2}$, it can be seen that

$$
\varepsilon_{2} \leq \phi_{2} \sum_{x=0}^{x_{0}-1}\left(\begin{array}{l}
n \\
x
\end{array}\right) r_{2} x\left(1-\gamma_{2}\right)^{n-x}
$$

where $\gamma_{2}$ is the value of $\gamma$ when $\zeta=\zeta_{0}-\delta^{*}$.

To illustrate the above inequalities a situation similar to the one described in the second example of the previous section is considered. In particular, suppose that $k=3, \zeta_{0}=.8,0 \leqslant \alpha \leqslant .1$, and $.15 \leqslant \beta$ $\leqslant .3$. Further suppose that $\mu$ and $\sigma^{2}$ are estimated to be .75 and .10 , respectively. Thus, $\tau_{1}=.7, m_{1}=$ $.0125, \phi_{1}=.417, \tau_{2}=.9, m_{2}=.1125$, and $\phi_{2}=.4$. Hence,

$$
\varepsilon_{1} \leq .417 \sum_{x=x_{0}}^{n}\left(\begin{array}{l}
n \\
x
\end{array}\right) .7648^{x} \cdot 2352^{n-x}
$$

and

$$
\varepsilon_{2} \leq .4 \sum_{x=0}^{x_{0}-1}\left(\begin{array}{l}
n \\
x
\end{array}\right) \cdot 88^{x} \cdot 12^{n-x} .
$$


The smallest number of skills so that simultaneously $\varepsilon_{1} \leqslant .1$ and $\varepsilon_{2} \leqslant .1$ is $n=59$.

\section{Concluding Remarks}

This paper has examined some of the problems that occur when using the proficiency tests currently being developed by many school systems. It is evident that more investigations need to be made. As previously indicated, better methods are needed for determining the optimal number of distractors per multiple-choice item and the optimal number of items per skill.

It has been argued that in terms of measuring achievement, a test should be constructed using an item-sampling principle (e.g., Harris, Pearlman, \& Wilcox, 1977). The author's experience with people constructing proficiency tests is that this approach is, indeed, used in many cases. However, there is also the problem that frequently a test does not consist of a random sample of skills, but rather skills are selected because they are judged to be the most important of those available. In this case, the efficacy of using the test length solution presented here might be in doubt. Alternatively, proficiency might be defined in terms of a hypothetical domain of skills where only the most important skills are represented in the item pool. In this case an item-sampling view of the test might be acceptable, and so the test length solution can be applied. It is noted that arbitrarily imposing the binomial error model has yielded good results using real data for certain measurement problems (e.g., Keats \& Lord, 1962; Lord, 1965; Subkoviak, 1978) but that in terms of test length the extent to which good results are obtained is not clear.

Another important point is that the test length solution is highly sensitive to the values of $\alpha$ and $\beta$. As was demonstrated, if completion items are used and $\beta=0$, a reasonably small number of items might be required even when the conservative solution to determining test length is applied. In many situations, there is the practical difficulty of physically scoring completion items, and so multiplechoice items are typically used. Accordingly, it would be beneficial to have some procedure that corrects for the errors $\alpha$ and $\beta$ in such a way that not too many multiple-choice items would be needed to ensure a reasonably high probability of making a correct decision for an examinee. For example, the usual correction-for-guessing formula score, which assumes guessing is at random, might be used. In many cases guessing is not at random, but perhaps this approach will still require fewer items than would otherwise be needed. Several other possibilities are currently being investigated; the results will appear in a forthcoming paper.

\section{References}

Anderson, T. W. On estimation of parameters in latent structure analysis. Psychometrika. 1954, 19 . $1-10$.

Brownless, V. T., \& Keats, J. A. A retest method of studying partial knowledge and other factors influencing item response. Psychometrika, 1958, 23. $67-73$.

Fhanér, S. Item sampling and decision making in achievement testing. British Journal of Mathematical and Statistical Psychology. 1974, 27. 172-175.

Formann, A. K. A note on parameter estimation for Lazarsfeld's latent class analysis. Psychometrika. 1978. 43, 123-126.
Gibbons, J., Olkin, I., \& Sobel, M. Selecting and ordering populations: $A$ new statistical methodology. New York: John Wiley, 1977.

Green, B. F. A general solution for the latent class model of latent structure analysis. Psychometrika, 1951, 16. 151-166.

Goodman, L. A. Exploratory latent structure analysis using both identifiable and unidentifiable models. Biometrika, 1974, 61, 215-231.

Harper, D. Local dependence latent structure models. Psychometrika, 1972, 37, 53-59.

Harris, C. W. Some technical characteristics of mastery tests. In C. W. Harris, M. C. Alkin, \& W. J. Popham (Eds.), Problems in criterion-ref- 
erenced measurement (CSE Monograph Series in Evaluation No. 3). Los Angeles: University of California, Center for the Study of Evaluation, 1974.

Harris, C. W., Pearlman, A. P., \& Wilcox, R. R. Achievement test items-Methods of study (CSE Monograph Series in Evaluation No. 6). Los Angeles: University of California, Center for the Study of Evaluation, 1977.

Huynh, H. Statistical consideration of mastery scores. Psychometrika, 1976, 41, 65-78.

Keats, J. A. \& Lord, F. M. A theoretical distribution for mental test scores. Psychometrika, 1962, 27, 59-72.

Knapp, T. R. The reliability of a dichotomous test item: A "correlationless" approach. Journal of Educational Measurement, 1977, 14, 237-252.

Lazarsfeld, P. F., \& Henry, N. W. Latent structure analysis. New York: Houghton Mifflin, 1968.

Lord. F. M. A strong true-score theory, with applications. Psychometrika, 1965, 30, 239-270.

Lord. F. M. Optimal number of choices per item-a comparison of four approaches. Journal of Educational Measurement. 1977, 14, 33-38.

Lord, F. M., \& Novick, M. R. Statistical theories of mental test scores. Reading, MA: AddisonWesley, 1968.

Macready, G. B., \& Dayton, C. M. The use of probabilistic models in the assessment of mastery. Journal of Educational Statistics, 1977, 2, 99-120.

Marks, E., \& Noll, G. A. Procedures and criteria for evaluating reading and listening comprehension tests. Educational and Psychological Measurement, 1967, 27, 335-348.

Novick, M. R., \& Lewis, C. Prescribing test length for criterion-referenced measurement. In $\mathrm{C}$. W. Harris, M. C. Alkin, \& W. J. Popham (Eds.), Problems in criterion-referenced measurement (CSE Monograph Series in Evaluation No. 3). Los Angeles: University of California, Center for the Study of Evaluation, 1974.
Skibinsky, M. The maximum probability of an interval when the mean and variance are known. Sankhya, 1977, Series A, 39, 144-159.

Subkoviak, M. J. Empirical investigation of procedures for estimating reliability for mastery tests. Journal of Educational Measurement, 1978, 15, 111-116.

Wilcox, R. R. Estimating the likelihood of a falsepositive or false-negative decision with a mastery test: An empirical Bayes approach. Journal of Educational Statistics, 1977, 2, 289-307.

Wilcox, R. R. Applying ranking and selection techniques to determine the length of a mastery test. Educational and Psychological Measurement, 1979, 31, 13-22. (a)

Wilcox, R. R. Achievement tests and latent structure models. British Journal of Mathematical and Statistical Psychology, 1979, 32, 61-71. (b)

Wilcox, R. R. On false-positive and false-negative decisions with a mastery test. Journal of Educational Statistics, 1979, 4, 59-73. (c)

\section{Acknowledgments}

The work upon which this publication is based was performed pursuant to a grant (contract) with the $\mathrm{Na}$ tional Institute of Education, Department of Health, Education, and Welfare. Points of view or opinions stated do not necessarily represent official NIE position or policy.

\section{Author's Address}

Send requests for reprints or further information to Rand R. Wilcox, University of California, Center for the Study of Evaluation, UCLA Graduate School of Education, 145 Moore Hall, Los Angeles, CA 90024. 